\title{
Stochastic Resonance Effects Reveal the Neural Mechanisms of Transcranial Magnetic Stimulation
}

\author{
Dietrich Samuel Schwarzkopf, ${ }^{1,2}$ Juha Silvanto, ${ }^{3,4}$ and Geraint Rees ${ }^{1,2}$ \\ ${ }^{1}$ UCL Institute of Cognitive Neuroscience, University College London, London WC1N 3AR, United Kingdom, ${ }^{2}$ Wellcome Trust Centre for Neuroimaging at \\ UCL, London WC1N 3BG, United Kingdom, and ${ }^{3}$ Brain Research Unit, Low Temperature Laboratory and ${ }^{4}$ Advanced Magnetic Imaging Centre, School of \\ Science and Technology, Aalto University, FI-00076 Espoo, Finland
}

Transcranial magnetic stimulation (TMS) is a popular method for studying causal relationships between neural activity and behavior. However, its mode of action remains controversial, and so far there is no framework to explain its wide range of facilitatory and inhibitory behavioral effects. While some theoretical accounts suggest that TMS suppresses neuronal processing, other competing accounts propose that the effects of TMS result from the addition of noise to neuronal processing. Here we exploited the stochastic resonance phenomenon to distinguish these theoretical accounts and determine how TMS affects neuronal processing. Specifically, we showed that online TMS can induce stochastic resonance in the human brain. At low intensity, TMS facilitated the detection of weak motion signals, but with higher TMS intensities and stronger motion signals, we found only impairment in detection. These findings suggest that TMS acts by adding noise to neuronal processing, at least in an online TMS protocol. Importantly, such stochastic resonance effects may also explain why TMS parameters that under normal circumstances impair behavior can induce behavioral facilitations when the stimulated area is in an adapted or suppressed state.

\section{Introduction}

Transcranial magnetic stimulation (TMS) has become a ubiquitous tool in cognitive neuroscience due to its ability to reveal causal roles of neuronal processing in different cortical areas in the human brain [e.g., Walsh and Pascual-Leone (2003), Cowey (2005), and Chambers and Mattingley (2005)]. However, the mechanisms by which it affects neuronal activity remain poorly understood, limiting the interpretation of many TMS studies. Traditionally, TMS effects are referred to as "virtual lesions" (e.g., Walsh and Pascual-Leone, 2003), suggesting that TMS is akin to inducing a brain lesion in a neurologically normal observer through transient suppression of neuronal processing. However, there are many findings from TMS experiments that do not fit such a simplistic conceptualization: for example, TMS stimulation parameters that normally disrupt motion detection can actually have facilitatory effects if the targeted area is in a suppressed, abnormal, or adapted state (Silvanto et al., 2007, 2008; Thompson et al., 2008; Siebner et al., 2009). Furthermore, while some accounts propose that TMS modulates behavior by suppressing neuronal signals (Harris et al., 2008), other effects have been accounted for by instead postulating that TMS adds or induces neural noise (Walsh and Pascual-Leone, 2003; Silvanto and Muggleton, 2008; Ruzzoli et al., 2010). It remains unclear which of these accounts is correct, or whether there is a single

Received Sept. 16, 2010; revised 0ct. 16, 2010; accepted Nov. 7, 2010.

G.R. and D.S.S. were funded by the Wellcome Trust. J.S. was funded by a Royal Society International Travel Grant.

Correspondence should be addressed to Dietrich Samuel Schwarzkopf, Wellcome Trust Centre for Neuroimaging

at UCL, London WC1N 3BG, UK. E-mail: s.schwarzkopf@fil.ion.ucl.ac.uk.

DOI:10.1523/JNEUROSCI.4863-10.2011

Copyright $\odot 2011$ the authors $\quad 0270-6474 / 11 / 313143-05 \$ 15.00 / 0$ unified account that can explain the wide range of behavioral TMS effects.

Here we addressed this question by exploiting the stochastic resonance phenomenon (Stocks, 2000). Stochastic resonance describes a phenomenon occurring in man-made or natural systems that have a measurement threshold. In such a system, information transfer is enhanced in a nonlinear manner by the injection of low levels of noise that effectively lower the response threshold of such a system. This is because without noise most of the signal does not pass the threshold for information transfer. Only the addition of optimal levels of noise pushes weak subthreshold signals across the threshold, improving information transfer. There is increasing evidence that stochastic resonance plays a role in sensory signal detection in the human brain ( $\mathrm{Si}$ monotto et al., 1997; Kitajo et al., 2003; Lugo et al., 2008; Sasaki et al., 2008). Consider the images in Figure 1. In the image at the top of the figure, the signal is weak and below threshold, rendering the image very difficult to identify. In the images in the middle and at the bottom, random luminance noise has been added to each pixel. While the high level of noise drowns out the signal in the bottom image, the middle image is actually the clearest of the three. This is because a low level of noise has been added, which pushes the intensity of some signal pixels above threshold, improving identification. Thus, the addition of low-level noise can effectively enhance the detection of a weak signal.

We reasoned that if online TMS introduced neuronal noise, the effects of stimulation should be characteristic of stochastic resonance: at low intensity, TMS should facilitate the behavioral detection of weak, but not strong, signals. Conversely, at high intensity, TMS should lead to disruption of behavioral performance for both weak and strong signals. Alternatively, if TMS 


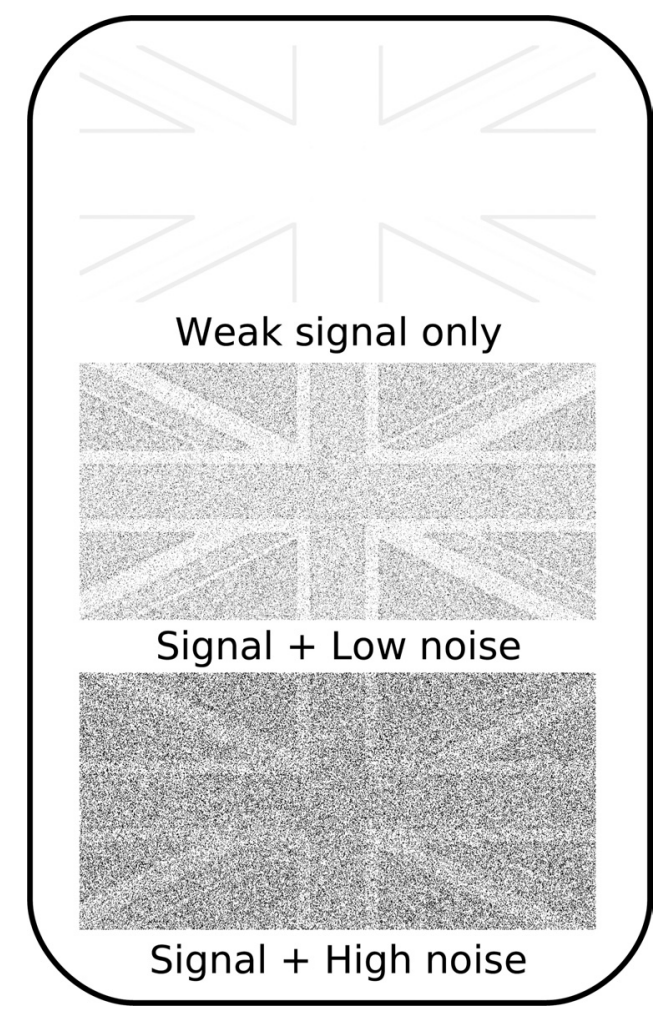

Figure 1. Stochastic resonance. Top, When the signal (picture of a flag) is weak, it is difficult to recognize the image, as only pixels at the edges of the differently colored regions pass the threshold. Bottom, Adding high levels of noise to the image drowns out the signal, and recognition remains difficult. Middle, Recognition can be best when an optimal amount of noise is added. This is because the noise pushes some signal pixels above the threshold.

instead suppressed neuronal activity, then impaired behavior is predicted at all TMS intensities, without facilitation for weak signals in the presence of low-intensity TMS. To anticipate our findings, we demonstrated that TMS produces effects on a standard motion direction discrimination task entirely consistent with stochastic resonance. This result can be interpreted as TMS injecting noise into the human visual system and thus suggests the mechanism by which TMS modulates behavior.

\section{Materials and Methods}

Participants. Ten healthy participants (6 males, aged 20-35, mean: 26) with normal or corrected-to-normal visual acuity took part in the experiment. We obtained written informed consent from all participants. The experiment was approved by the local ethics committee and participants were treated in accordance with the declaration of Helsinki.

Visual stimulus. All stimuli were presented centrally on an SVGA 17inch monitor set at a resolution of $1024 \times 768$ and a refresh rate of $100 \mathrm{~Hz}$ using E-Prime (Psychology Software Tools). Viewing distance was $57 \mathrm{~cm}$. The global motion stimulus consisted of 100 white dots (1 pixel each, luminance of white: $103 \mathrm{~cd} / \mathrm{m}^{2}$ ), placed at random positions within an imaginary square that subtended $4.3 \times 4.3$ degrees of visual angle. The coherent dots were moving either to the right or left within the virtual square on a black (luminance: $0.07 \mathrm{~cd} / \mathrm{m}^{2}$ ) background at a speed of 1 pixel per frame (at $4.3 \mathrm{deg} / \mathrm{s}$ ); within a trial noise dots changed direction randomly every $10 \mathrm{~ms}$. Each trial began with a fixation point appearing in the middle of the screen for $500 \mathrm{~ms}$, followed by a blank screen for 500 $\mathrm{ms}$, after which the stimulus appeared. Stimulus duration was between 40 and $70 \mathrm{~ms}$, depending on participant's ability (4-7 frames lasting for $10 \mathrm{~ms}$ each). After each trial, a blank screen appeared for $1000 \mathrm{~ms}$ before the start of the next trial.

Thresholding. We determined motion coherence thresholds for two levels of performance in each participant: $60 \%$ and $85 \%$ correct. This was achieved by running a block in which motion coherence (i.e., the amount of dots moving coherently either to the left or right) ranged from 0 to 100 with eight levels (with 20 trials per level with the method of constant stimuli, i.e., coherence levels were randomly interleaved). A logistic psychometric curve was then fit to the data of each individual participant using the maximum likelihood procedure implemented in psignifit (Wichmann and Hill, 2001a,b), a freely available toolbox for MATLAB (MathWorks). We then extrapolated the two threshold levels of motion coherence from the psychometric function. Initially, the motion stimulus contained six frames. If performance was such that it did not allow fitting for these two points of accuracy (i.e., the stimulus was too easy or too difficult), it was made easier/more difficult by addition/removal of one frame. Five participants performed the task with four frames (average low-coherence threshold: $25 \%$; average high-coherence threshold: $68 \%$ ), two with five frames (low coherence: $36 \%$; high coherence: $87 \%$ ), two with six frames (low coherence: $58 \%$; high coherence: $82 \%$ ), and one with seven frames (low coherence: $43 \%$; high coherence: 100\%).

Transcranial magnetic stimulation. We delivered TMS using a Magstim Super Rapid machine with a $70 \mathrm{~mm}$ figure-of-eight coil. We located the motion-sensitive complex V5/MT using the functional method of inducing moving phosphenes (Stewart et al., 1999; for detailed discussion, see Walsh and Pascual-Leone, 2003), a technique that has been used in a number of studies on V5/MT function [e.g., Pascual-Leone and Walsh (2001) and Campana et al. (2002, 2006)] and that corresponds well with localization by functional magnetic resonance imaging (Thompson et al., 2009). The mean scalp coordinates were $3.2 \mathrm{~cm}$ dorsal and $5.4 \mathrm{~cm}$ lateral from the inion. We stimulated V5/MT in the left hemisphere in all participants because it has consistently been found to produce phosphenes more reliably than the right hemisphere (Beckers and Hömberg, 1992; Stewart et al., 1999; Antal et al., 2001). We determined the threshold TMS intensity for inducing phosphenes in each participant. Mean phosphene threshold with triple-pulse TMS measured using the binary search paradigm (Tyrell and Owens, 1988$)$ was $53 \%( \pm 7.6 \%$ SD) of the maximum stimulator output.

In the main experiment, on each trial, we applied triple-pulse TMS (applied at $20 \mathrm{~Hz}$; i.e., pulse gap of $50 \mathrm{~ms}$ ) immediately after the offset of the motion stimulus. These stimulation parameters are the same as used in a previous TMS study on V5/MT function (Silvanto et al., 2008). We applied TMS at three different levels: $60 \%, 80 \%$, and $100 \%$ of the phosphene threshold of each individual participant. In addition, a no-TMS condition was carried out. In the no-TMS condition, the TMS coil was held perpendicular against the scalp, such that one wing of the coil touched the scalp location of V5/MT, with the stimulation intensity set at $60 \%$ of the phosphene threshold. The no-TMS condition thus controls for the auditory artifact induced by TMS.

Procedure. At the beginning of the first session, we localized each participant's V5/MT region. In all subsequent experiments, participants were required to report whether the stimulus moved to the left or right (Fig. 2A). Initially, participants were given practice blocks with the motion discrimination task. They were then thresholded for the two levels of performance: low coherence ( $60 \%$ correct) and high coherence $(85 \%$ correct).

The actual experiment was divided into eight blocks, i.e., two blocks for each of the four TMS conditions: no TMS, low TMS (60\% of phosphene threshold), medium TMS (80\%), and high TMS (100\%). Each block consisted of 72 trials; of these, 36 were of the low coherence and 36 of the high coherence. Half of the trials contained leftward motion, and the other half contained rightward motion. There were thus a total of 72 trials for both the low-coherence and high-coherence targets for each TMS condition. The first half of the experiment (i.e., the first four blocks) contained one block of each of the four TMS conditions; the order of these was randomly assigned. The order of the second half of the experiment was the reverse of the first half.

\section{Results}

In this study, we investigated whether TMS effects exhibit stochastic resonance in their interaction with visual motion detection. In each individual participant, we first determined the motion coherence thresholds that afforded low and high discrim- 
A
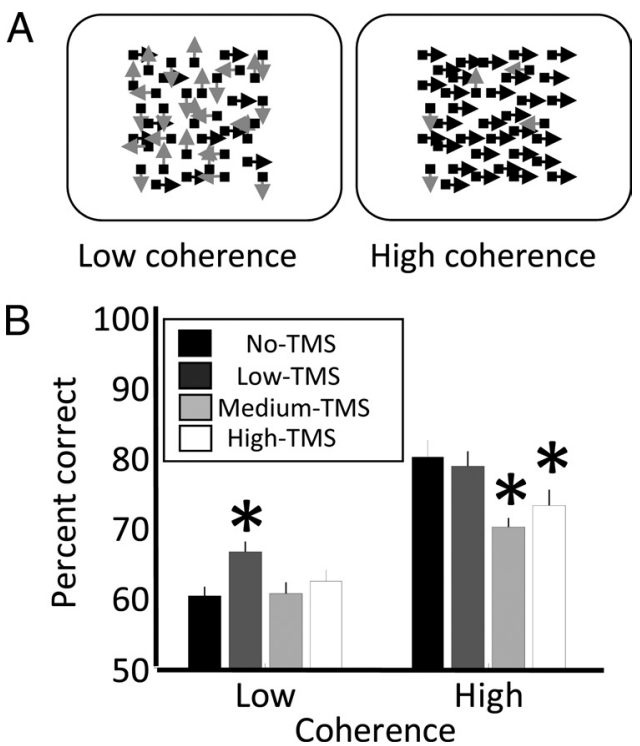

Figure 2. $\quad A, 0$ n each trial, participants were instructed to discriminate the direction of motion of a random dot stimulus where a proportion of dots moved either left or right (black arrows), while the direction of remaining dots was random (gray arrows). We tested two levels of coherence affording $60 \%$ and $85 \%$ correct (left and right panels), respectively. TMS was delivered simultaneously with stimulus onset at one of three intensities or not at all. $\boldsymbol{B}$, Percentage correct discrimination plotted for the two coherence levels and the four levels of TMS intensity. The asterisks above a bar indicate that performance in this condition was significantly (two-tailed $t$ test $p<0.05$ ) different from trials without TMS (black bars).

ination performance (60\% and $85 \%$ correct, respectively) (Fig. $2 A$ ) on a motion direction discrimination task. We then tested the effects of TMS with varying intensity over the motionsensitive complex V5/MT on the behavioral performance at these two levels.

Figure $2 B$ plots percentage correct discrimination for the two levels of motion coherence (low and high) and the four levels of TMS intensity (no, low, medium, and high). As expected, discrimination performance for the high-coherence stimuli was significantly greater than for the low coherence (two-way repeated-measures ANOVA: $F_{(1,9)}=35.08, p=$ $0.0002)$. Specifically, performance in the no-TMS condition was $60 \%$ for the low coherence and $80 \%$ for the high coherence, confirming the reliability of our initial thresholding procedure, as these accuracies were not significantly different from what would be expected from the thresholding (low coherence: $t_{(9)}=0.16$, $p=0.88$; high coherence: $\left.t_{(9)}=-1.8, p=0.105\right)$. Furthermore, we found that TMS significantly modulated behavior $\left(F_{(2.3,21)}=\right.$ 7.22, $p=0.003$, Greenhouse-Geisser correction: $\varepsilon=0.77$ ).

Crucially, however, our results revealed an interaction between TMS intensity and the coherence level $\left(F_{(2.3,21)}=4.47, p=\right.$ $0.02, \varepsilon=0.77)$. At the high coherence level, TMS significantly reduced discrimination performance relative to the no-TMS condition for the medium $\left(t_{(9)}=3.4, p=0.008\right)$ and the high $\left(t_{(9)}=2.3, p=0.047\right)$ intensity of TMS, but not for the low intensity $\left(t_{(9)}=0.48, p=0.641\right)$. In contrast, at the low coherence level, performance was significantly increased by $\sim 7 \%$ relative to trials without TMS for low TMS intensity only $\left(t_{(9)}=-3.63, p=\right.$ 0.006 ). For higher TMS intensities, there was no change (medium TMS: $t_{(9)}=-0.12, p=0.904$; high TMS: $t_{(9)}=-0.9, p=0.389$ ).

Interestingly, at both levels of coherence, discrimination performance appeared to be slightly greater for high-intensity than for medium-intensity TMS. However, even when pooling the data across coherence levels, the performances were $65.2 \%$ and $67.7 \%$ for medium- and high-intensity TMS, respectively, a difference that was not significant $\left(t_{(9)}=-1.68, p=0.128\right)$. Performance levels for individual participants are shown in supplemental Table S1 (available at www.jneurosci.org as supplemental material).

\section{Discussion}

Our results demonstrate that TMS effects are characteristic of stochastic resonance. While high-intensity TMS disrupted performance for discriminating the direction of clearly distinguishable high-coherence random dot stimuli, at low intensity, TMS facilitated the discrimination of low-coherence motion signals relative to no TMS. The results of our study are incompatible with a strong suppression account and can instead be interpreted as evidence that online TMS injects neuronal noise. If TMS uniformly suppressed neuronal signals, then it would have disrupted behavioral performance at all levels of coherence, a pattern that was not observed. Instead, our finding of facilitation only when low coherence and low TMS intensity stimulation were combined shows that this was not the case.

In TMS experiments on perception, there are typically a number of potential confounds that may account for some of the results. In the present study, it might be argued that the disruptive effect of high-intensity TMS was due to TMS inducing phosphenes that distracted perception of the motion stimulus. However, even though in the high-intensity condition TMS was applied at phosphene threshold, the frequency of phosphene induction is much less frequent when the subject is not attending to the phosphene (Bestmann et al., 2007). Furthermore, participants were asked to report if they detected phosphenes during the experiment and none did so. It is thus unlikely that phosphene induction can explain the disruptive effect of TMS on highcoherence stimuli. A second conceivable confound is that highintensity TMS for high-coherence targets induces more blinking than low-intensity TMS. However, great care was taken that blinking was not induced by TMS through accurate coil placement and direct observation of the participants by the experimenter. Furthermore, one could argue that there was a bias in the likelihood of inducing suppressive versus facilitative effects for detection of weak ( $60 \%$ threshold) versus strong ( $80 \%$ threshold) signals. However, none of these nonspecific effects can explain the key finding in the present study, namely the facilitatory effect of low-intensity TMS (but not high-intensity TMS) on lowcoherence targets. It is also important to note that the present findings may only apply to online TMS protocols (where TMS is applied at the time of presentation of the stimuli), and different mechanisms may underlie the effects found in offline TMS paradigms (where TMS is applied before stimulus presentation) such as theta burst TMS and $1 \mathrm{~Hz}$ TMS.

What is the nature of the neuronal noise added by TMS? Perhaps the most parsimonious account is that by exciting neuronal populations tuned to a wide range of different directions of motion, TMS increases the uncertainty about the global direction in the stimulus. Conceptually, one might regard this as perceived deviation in the direction each of the dots was moving. At low TMS intensity, this may not be severe enough to alter the perceived direction of signal dots-but it is sufficient to push the perceived direction of some noise dots to sum with the signal dots and thus enhance behavioral performance. Conversely, for the high-coherence stimuli, high-intensity TMS will cause a deterioration of performance because some of the signal dots are no longer seen as moving in the signal direction. 
An alternative account for the pattern of results we observed would entail that the direction of TMS effects differs between stimulation intensities, such that TMS at high intensity suppresses neuronal activity but that it boosts the signal at low intensities. There is some electrophysiological evidence consistent with this (Funke and Benali, 2010). Moliadze et al. (2003) showed that when applied concurrently with visually induced neural activity, low-intensity TMS facilitated neural firing, whereas highintensity TMS had a suppressive effect. However, critically, such an account does not change the explanation for the facilitatory effect observed with low-intensity TMS. TMS is likely to affect all neuronal populations within the stimulated brain area. Thus, low-intensity TMS would excite neurons tuned to all directions. This effectively is the same as adding noise to the direction signal. This is not inconsistent with the electrophysiological and behavioral evidence that TMS effects may also depend on the current state of activity in the stimulated neurons [e.g., Moliadze et al. (2003), Silvanto and Muggleton (2008), Pasley et al. (2009), and Siebner et al. (2009)].

We did not observe a deterioration of performance for the low-coherence stimuli when applying higher TMS intensities. Instead, performance remained at the $60 \%$ correct baseline. This may be somewhat surprising when one considers that mediumand high-intensity TMS for the high-coherence stimuli disrupted discrimination performance. However, a decline of performance for these conditions would be expected if TMS suppressed the neuronal signal. Therefore, we believe this result is further evidence consistent with the account that TMS injects neuronal noise.

Stochastic resonance is a phenomenon whereby signal transmission in a threshold measurement system is enhanced by the addition of low levels of noise (Stocks, 2000). Previous psychophysical studies posited that stochastic resonance optimizes signal detection in the human visual system through internal noise (Simonotto et al., 1997; Kitajo et al., 2003). Our results contribute to this work by suggesting that TMS manipulates the level of internal noise. We further suggest a reason for the conflicting effects of TMS observed by previous studies. When signals are weak, TMS may facilitate behavior by adding low levels of noise. Future work should explore whether the noise induced by TMS is stationary or varies across time.

The mechanisms discussed may provide a useful general framework for TMS effects, as they can explain why TMS can induce both behavioral disruptions and facilitations. For example, it may explain findings that TMS facilitates the detection of stimuli detected by neuronal populations that have been subject to neuronal adaptation (Silvanto et al., 2007; L. Cattaneo et al., 2010; Z. Cattaneo et al., 2010; Cohen Kadosh et al., 2010), even though the same TMS parameters in the absence of adaptation impair performance. In the context of stochastic resonance effects, there are two relevant differences that result from adaptation and the reduction in neuronal excitability that it induces. First, presentation of the target stimulus induces a lower level of activation, and thus the ability to detect the target stimulus is reduced (akin to making the stimulus more difficult). Second, TMS is less effective in activating the neurons, as the neurons are less susceptible after adaptation; this is akin to reducing the TMS intensity. Therefore, the facilitatory effect of TMS when applied after adaptation may be due to adaptation having a similar effect to that caused by reducing the TMS intensity and increasing the difficulty of the target stimulus-thus inducing conditions in which stochastic resonance is observed. The same explanation may apply to the facilitatory effect of TMS when the targeted region is in a suppressed state (Silvanto et al., 2008). While this question requires further study, stochastic resonance may provide one explanation of online TMS effects in a wide range of paradigms in which facilitatory effects are observed. Furthermore, the present results do not rule out the possibility that TMSinduced behavioral facilitations and impairments can result from state-dependent neural effects [for example, suppression of neural processing in highly active neurons, and enhancement of processing in less active neural populations (e.g., Moliadze et al., 2003)].

To date, it has remained controversial whether TMS acts by introducing neuronal noise or by suppressing activity, with evidence in support of both views (Harris et al., 2008; Ruzzoli et al., 2010). The advantage of using predictions derived from stochastic resonance when investigating this question is that such effects can be explained in terms of noise, and it thus provides compelling evidence in support of the "noise" explanation of TMS effects.

\section{References}

Antal A, Nitsche MA, Paulus W (2001) External modulation of visual perception in humans. Neuroreport 12:3553-3555.

Beckers G, Hömberg V (1992) Cerebral visual motion blindness: transitory akinetopsia induced by transcranial magnetic stimulation of human area V5. Proc Biol Sci 249:173-178.

Bestmann S, Ruff CC, Blakemore C, Driver J, Thilo KV (2007) Spatial attention changes excitability of human visual cortex to direct stimulation. Curr Biol 17:134-139.

Campana G, Cowey A, Walsh V (2002) Priming of motion direction and area V5/MT: a test of perceptual memory. Cereb Cortex 12:663-669.

Campana G, Cowey A, Walsh V (2006) Visual area V5/MT remembers "what" but not "where". Cereb Cortex 16:1766-1770.

Cattaneo L, Sandrini M, Schwarzbach J (2010) State-dependent TMS reveals a hierarchical representation of observed acts in the temporal, parietal, and premotor cortices. Cereb Cortex 20:2252-2258.

Cattaneo Z, Devlin JT, Salvini F, Vecchi T, Silvanto J (2010) The causal role of category-specific neuronal representations in the left ventral premotor cortex (PMv) in semantic processing. Neuroimage 49:2728-2734

Cohen Kadosh R, Muggleton N, Silvanto J, Walsh V (2010) Double dissociation of format-dependent and number-specific neurons in human parietal cortex. Cereb Cortex 20:2166-2171.

Cowey A (2005) The Ferrier Lecture 2004: what can transcranial magnetic stimulation tell us about how the brain works? Philos Trans R Soc Lond B Biol Sci 360:1185-1205.

Funke K, Benali A (2010) Cortical cellular actions of transcranial magnetic stimulation. Restor Neurol Neurosci 28:399-417.

Harris JA, Clifford CWG, Miniussi C (2008) The functional effect of transcranial magnetic stimulation: signal suppression or neural noise generation? J Cogn Neurosci 20:734-740.

Kitajo K, Nozaki D, Ward LM, Yamamoto Y (2003) Behavioral stochastic resonance within the human brain. Phys Rev Lett 90:218103.

Lugo E, Doti R, Faubert J (2008) Ubiquitous crossmodal stochastic resonance in humans: auditory noise facilitates tactile, visual and proprioceptive sensations. PLoS ONE 3:e2860.

Moliadze V, Zhao Y, Eysel U, Funke K (2003) Effect of transcranial magnetic stimulation on single-unit activity in the cat primary visual cortex. J Physiol 553:665-679.

Pascual-Leone A, Walsh V (2001) Fast backprojections from the motion to the primary visual area necessary for visual awareness. Science 292:510-512.

Pasley BN, Allen EA, Freeman RD (2009) State-dependent variability of neuronal responses to transcranial magnetic stimulation of the visual cortex. Neuron 62:291-303.

Ruzzoli M, Marzi CA, Miniussi C (2010) The neural mechanisms of the effects of transcranial magnetic stimulation on perception. J Neurophysiol 103:2982-2989.

Sasaki H, Sakane S, Ishida T, Todorokihara M, Kitamura T, Aoki R (2008) Suprathreshold stochastic resonance in visual signal detection. Behav Brain Res 193:152-155.

Siebner HR, Hartwigsen G, Kassuba T, Rothwell JC (2009) How does trans- 
cranial magnetic stimulation modify neuronal activity in the brain? Implications for studies of cognition. Cortex 45:1035-1042.

Silvanto J, Muggleton NG (2008) New light through old windows: moving beyond the "virtual lesion" approach to transcranial magnetic stimulation. Neuroimage 39:549-552.

Silvanto J, Muggleton NG, Cowey A, Walsh V (2007) Neural adaptation reveals state-dependent effects of transcranial magnetic stimulation. Eur J Neurosci 25:1874-1881.

Silvanto J, Cattaneo Z, Battelli L, Pascual-Leone A (2008) Baseline cortical excitability determines whether TMS disrupts or facilitates behavior. J Neurophysiol 99:2725-2730.

Simonotto E, Riani M, Seife C, Roberts M, Twitty J, Moss F (1997) Visual perception of stochastic resonance. Phys Rev Lett 78:1186-1189.

Stewart L, Battelli L, Walsh V, Cowey A (1999) Motion perception and perceptual learning studied by magnetic stimulation. Electroencephalogr Clin Neurophysiol Suppl 51:334-350.

Stocks NG (2000) Suprathreshold stochastic resonance in multilevel threshold systems. Phys Rev Lett 84:2310-2313.
Thompson B, Mansouri B, Koski L, Hess RF (2008) Brain plasticity in the adult: modulation of function in amblyopia with rTMS. Curr Biol 18:1067-1071.

Thompson B, Aaen-Stockdale C, Koski L, Hess RF (2009) A double dissociation between striate and extrastriate visual cortex for pattern motion perception revealed using rTMS. Hum Brain Mapp 30: 3115-3126.

Tyrell RA, Owens DA (1988) A rapid technique to assess the resting states of the eye and other threshold phenomena: the modified binary search (MOBS). Behav Res Methods Instrum Comput 20:137-141.

Walsh V, Pascual-Leone A (2003) Neurochronometrics of mind: transcranial magnetic stimulation in cognitive science. Cambridge, MA: MIT Press.

Wichmann FA, Hill NJ (2001a) The psychometric function: I. Fitting, sampling, and goodness of fit. Percept Psychophys 63:1293-1313.

Wichmann FA, Hill NJ (2001b) The psychometric function: II. Bootstrapbased confidence intervals and sampling. Percept Psychophys 63:13141329 